\title{
Use of accelerometers in the control of practical prosthetic arms
}

\author{
Peter J Kyberd and Adrian Poulton
}

\begin{abstract}
Accelerometers can be used to augment the control of powered prosthetic arms. They can detect the orientation of the joint and limb and the controller can correct for the amount of torque required to move the limb. They can also be used to create a platform, with a fixed orientation relative to gravity for the object held in the hand. This paper describes three applications for this technology, in a powered wrist and powered arm. By adding sensors to the arm making these data available to the controller, the input from the user can be made simpler. The operator will not need to correct for changes in orientation of their body as they move. Two examples of the correction for orientation against gravity are described and an example of the system designed for use by a patient. The controller for all examples is a distributed set of microcontrollers, one node for each joint, linked with the Control Area Network (CAN) bus. The clinical arm uses a version of the Southampton Adaptive Manipulation Scheme to control the arm and hand. In this control form the user gives simpler input commands and leaves the detailed control of the arm to the controller
\end{abstract}

Index Terms- Artificial Limbs, Prosthetic limbs, Prosthetic hand, Accelerometers

\section{INTRODUCTION}

$T_{\text {in }}^{\text {he }}$ he natural system uses a variety of different information sources in the control of bodily motion [1]. Historically, prosthetic limbs have used a minimum of sources. This is because of constraints of practicality on the eventual device (such as mass, size and price). As consumer electronics have increased the availability of compact, cheap, low power sensors, it is now possible to conceive of incorporating such information sources into prosthetic limbs to assist the user in the control of the device. One such source of information is accelerometers, which can provide information about the orientation of limb segments.

Acceleration can be used to measure the motion of a body. While variations of some of the methods described

This work was supported in part Canadian Foundation for Innovation, Canada Institutes of Health Research, New Brunswick Innovation Foundation.

Peter $\mathbf{J}$ Kyberd is Chair in Rehabilitation Cybernetics and Head of the Engineeering Science Department University of GreenwichUK. E3B 5A3 (email: p.j.kyberd@gre.ac.uk).

Adrian Poulton is at the Open University, Milton Keynes, UK (e-mail: Adrian.Poulton@open.ac.uk). here have been used in robotics previously, they have only recently become practicable in clinical prosthetics . What remains to be fully developed is power of the motors, microprocessors and batteries to allow a system small enough to be incorporated in a practical prosthesis. The applications described here represent an intermediate stage to full integration of the sensors into arm systems, where the sensors and controllers are separate and some aspects of the control, standard in robotics, cannot be realised in a field ready prosthesis. This paper describes applications created and used with prosthesis systems that are in use clinically.

This paper will outline three applications of a tri-axial accelerometer to prosthetic arms.

The use of accelerations alone as the measure of movement has a fundamental limitation. To measure motion the signal must be integrated over time. As a result signal noise tends to accumulate as an error in the signal, causing drift. These problems are well understood and there are techniques to successfully reduce/remove the drift [2]. For example; the use of sensors to detect times of no acceleration and remove the accumulated offset. A packaged version of this system is an Inertial Measurement Unit (IMU), a combination of accelerometers, gyroscopes and sometimes magnetometers. Knowledge of orientation of the accelerometer through the measurements made by the magnetometers and the gyroscope allows the system to determine when the acceleration being measured is due to gravity alone. At this time, the drift can be subtracted from the signal and errors reduced. The advances in silicon technology mean that IMUs are now very small, cheap and widely used. The applications described in this paper, use the instantaneous information from accelerometers, so the problems of long term drift that would be significant in navigation systems are essentially avoided here.

Accelerometers have not previously been used in exoprosthetics. In 1980 Swain [3,4] suggested the use of accelerometers in a whole arm prosthesis to create an inertial platform for the hand, making transport of objects held in the hand simpler. This was part of the Southampton Arm project that aimed to use additional information about the held object and its disposition to 
simplify the control of a multi joint prosthetic arm. The natural system uses a hierarchy to control bodily movements. This solution uses the top level of the human Central Nervous System (CNS) for conscious control. Beneath this are the subconscious levels that coordinate information on the mass of the arm and target object, its orientation, tactile information and background experience of many years, to hold an object stably with minimum physical and conscious effort. What could be referred to as the Southampton Prosthetic Philosophy aimed to create a hierarchy in the device, with the low level control being left to the electronic controller $[3,5,6]$, (conceived in an era where transistors were still novel and unreliable, systems now use microprocessor controllers). Shoulder motion was used to target the motion of the end point of the arm (the hand), through motions in two dimensions, (the third being controlled by trunk sway) $[3,4]$. At the time, the prosthesis was too heavy to be worn and the electronics were not compact enough to be practical. Subsequent technological developments have allowed these ideas to be explored further [7].

\section{Prosthesis Control}

The use, instruction and control of prostheses is generally achieved by capturing some element of bodily motion; scapula abduction or muscle contraction (detected through electromyography), and mapping this to the motion of a joint in the prosthesis. Traditionally, multiple degrees of freedom had to be controlled serially, with the user having to switch between axes (with the concomitant reduction in speed of action and increase in cognitive effort). In principle, a greater number of control channels can be detected through pattern recognition of the muscle signals on the forearm. After many years of laboratory based studies, these ideas are now being used clinically [8]. In this arena is one application of accelerometers. Subtle differences in patterns of signals occur as the arm changes orientation and researchers have employed them to enhance accuracy [9].

Unfortunately, these techniques rely on sufficient numbers of muscles being intact in the residual limb for the resulting signals to be meaningful. Without surgical intervention, the number of channels can be limited. This surgery relocates the nerve from a more distal (and absent) body part to control denervated more proximal muscle [10]. This technique is growing in application, but represents an invasion that some limb absent persons may not want, or the trauma may have made such procedures impossible. For these individuals other solutions are necessary. While a conventional controller is slower and harder to use, the wearer may choose to apply some other bodily movement to compensate for the action, which is quicker. Unfortunately this is more likely to lead to over-use injuries [11].

Electromyography is a common control input. The signal is a noisy signal with frequencies over a kilohertz [12]. To make a useful signal, the raw EMG is rectified and smoothed (low pass filtered). This creates a slow moving voltage level (a few hertz) that can be used to select a state, or drive an axis with joint velocity or joint position control. Hands are generally velocity controlled, but major joints such as the elbow can be position controlled more effectively. Clearly there is a trade off between speed of the signal (and thus speed of the controlled axis) and smoothness and ease of control. To overcome some variability, the smoothed EMG channel has a threshold that the command has to exceed, before the axis is under voluntary control.

Another reason for users compensating for the limitations in their prostheses is the cognitive burden required to control the device. Controlling a natural arm is generally subconscious, but a prosthesis requires concentration. Prosthetic arms work without feedback into the CNS and offer only serial control of each joint. So control requires conscious effort and visual concentration [13]. In these applications some of the low level control (such maintaining the orientation of the arm) can be managed by a microprocessor controller freeing the user from low level control burden. A user of a conventional prosthesis may employ humeral abduction rather than pro/supination of the wrist simply because it is quicker and easier to do. So although they might risk over-use injuries, they will tend to use compensations.

The Southampton Prosthetic Philosophy aimed to assist in the selection of the motion or axis and so lower the cognitive overhead in controlling the arm and the number of inputs needed to control a multi-axis arm. In the case in this paper, accelerometers are used to lower the cognitive burden of controlling the arm.

There are three ways that the accelerometer data can be used to assist control of the motions of the arm segments (shown in Figure 1):

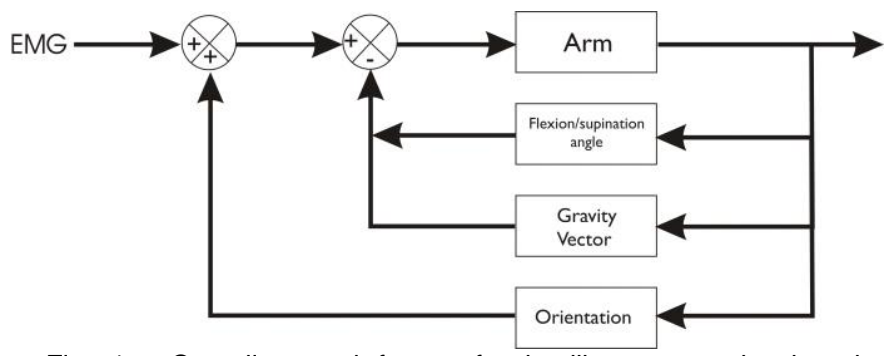

Fig. 1. Overall control format for intelligent arm showing the different examples of control made possible through use of accelerometer inputs.

The three forms of compensation the accelerometer can be used for, summarized in Figure 2: Segment orientation, where the orientation of the segment has an impact on the current required to move at a given pace. Segment Motion Compensation, where the segment 
remains in the same orientation relative to gravity, despite changes in the live segment, more proximal to the prosthesis. Inertial Platform, fixes the orientation of the object held in the hand of a whole arm prosthesis (shoulder, elbow, wrist).

\section{A. Segment orientation}

The most basic application of acceleration data is to use it to determine the orientation of a segment of the prosthesis. For example; the angle the forearm makes relative to gravity in an elbow prosthesis. As an elbow flexes from the horizontal the gravity vector accelerating the arm reduces as the sine of the angle with the vertical, this needs to be allowed for in a controller.

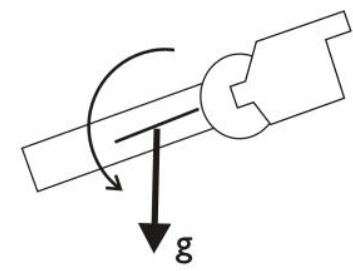

(a) Segment orientation

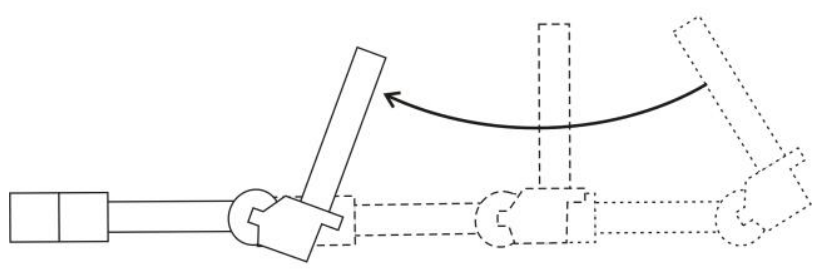

(b) Motion Compensation

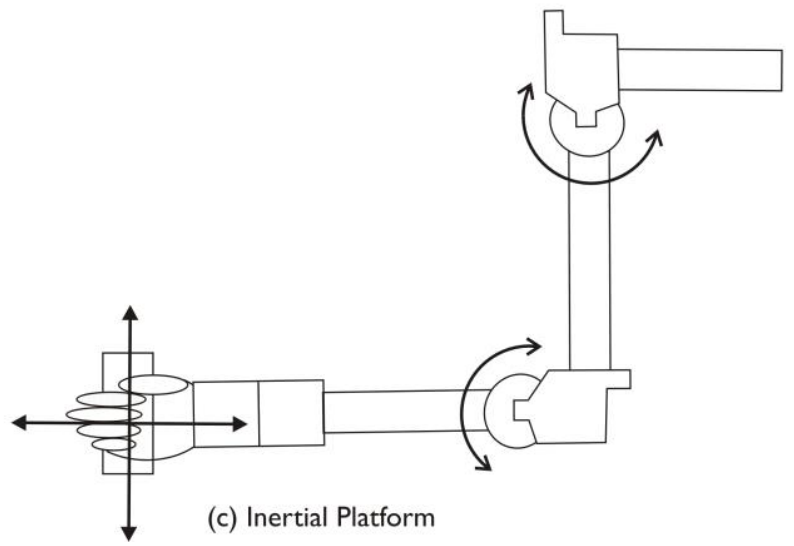

Fig. 2. Three forms of compensation by using the data from accelerometers Segment orientation - adjusts the motor demand based on the segments orientation relative to gravity. Motion Compensation - adjusts the orientation of a prosthetic segment, based on the movement of the remnant limb segment proximal of the prosthesis. Inertial Platform - maintains the

In a conventional arm prosthesis, the muscular demand (level of EMG contraction) is mapped directly to the voltage on the motor, then flexion speed is roughly linked to perceived contraction. However, clearly there is a difference between the demand for the elbow to move up against gravity and when it moves down with gravity. Thus the perceived effort to raise the hand will seem far greater than lowering it. While the natural system easily compensates for this, a prosthetic system will not. If the controller has information about the orientation of the arm it can match the thresholds for movement in either direction, more closely to each other. This can be achieved through measuring the angle of the segment and adjusting the gain according to the sine of the angle the arm makes with the gravity vector. While velocity control can remove the uncertainty when the joint is moving, it is only becoming adopted in prosthetic systems now. The addition of joint position movement is still rare in clinical prosthetics systems. The method proposed here can be added to existing prosthesis without modifications to the basic hardware or motor control electronics. In addition joint velocity control cannot compensate for the threshold before which the arm would move.

The angle $\square \square \square$ that gravity makes with the forearm segment is defined in Figure 3. In the sagittal plane the gravity vector is calculated as:

$\theta=\arctan (Y / Z)$

Where $\square$ is the gravity angle, making the gravity correction $\mathrm{C}_{\mathrm{G}}$

$C_{G}=\sin \theta$

Thus joint rotational velocity demand $V_{D}$ is:

$V_{D}=E M G D e m a n d+\left(C_{G} \times G_{a}\right)$

Where $G_{a}$ is the constant of proportionality when the forearm is held out in the horizontal plane and is thus resisting the largest pull of gravity $\left(\square=90^{\circ}\right)$.

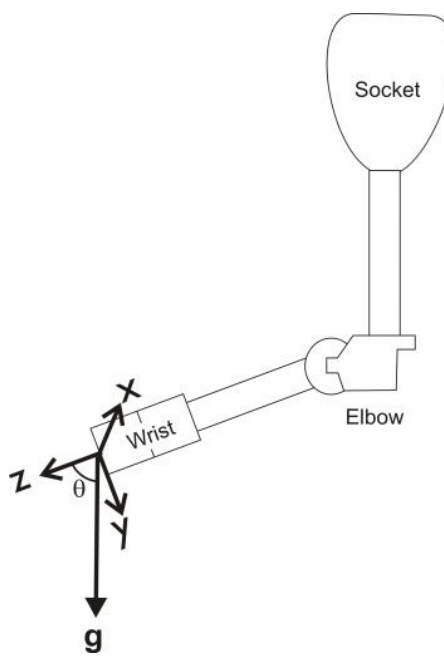

Fig. 3. Definition of arm orientation to gravity, showing key elements of the arms system and the three axis of the wrist mounted accelerometer. 


\section{B. Motion compensation}

If the prosthesis user has a loss above their elbow, but a working shoulder joint, when they wish to reach forward and keep their forearm level, (for example reaching forward to grasp an object on a table), they have to compensate by changing the flexion angle at the elbow. With a conventional system this level of control is challenging. The natural solution uses feedback of the position of the arm to control the orientation. A prosthesis user only has visual feedback, so moving these automatic control processes to the device reduces the cognitive overhead. Information from accelerometers can be used to maintain the angle the forearm segment makes with gravity. As the controller is instructed to maintain the level the gravity correction $\mathrm{C}_{\mathrm{G}}$ measured by the accelerometer is stored and used as the orientation demand, which is mapped to a motor velocity.

The controller uses the gravity vector to determine the arm's orientation. This takes into account if the arm is held in the conventional orientation or inverted against gravity (arm held over the head). The rest of the correction is a simple position control algorithm based on the difference between the recorded orientation relative to gravity and that detected by the accelerometer.

$V_{D}=\left(1+\mathrm{C}_{\mathrm{G}}\right) * \Delta$ Position $* \mathrm{G}_{\mathrm{m}}$

Where the Gain $\left(\mathrm{Gm}_{\mathrm{m}}\right)$ is the constant of proportionality and $\square$ Position is the difference in angular position to the target orientation.

In a practical system this feedback loop sits outside the previously described control so that the arm's response is uniform.

\section{Inertial Platform}

If an object is held in the hand of a full arm prosthesis (shoulder, elbow, wrist and hand), then any motion by the wearer is likely to change the orientation of the object (for example with a cup of liquid in their hand when walking). This motion can then be reduced using accelerometers based in the hand and on the shoulder harness of the user, to drive a third feedback path outside the previous two. In this case the accelerometer data can be used to generate an error signal for an inverse kinematic model of the arm. Thus the system creates an 'inertial platform'

Using these axes the arm can compensate for changes in orientation and position. As Swain [3] described; "to maintain constant arm and hand orientation during gross movements of the trunk", and to "maintain the orientation of the hand during feeding".

\section{EXAMPLES}

Different examples of these applications are described using an advanced prosthetic system; the ToMPAW arm $[7,14]$. This arm system was developed to investigate ideas in the control and application of upper limb prosthetic components. The first generation arm was the first prosthesis to be controlled using a local area network, and was first fitted to users in Europe in 1999. The advantage of the bus system is that it allows more sensors and systems to be added to the arm, and requires fewer wires running down the arm. This form of solution has proven successful and manufacturers have begun to adopt their own bus communications systems.

A later version of the ToMPAW arm was instrumented and a variant of the Swain shoulder joystick was used to control the arm in laboratory trials [7]. This consisted of an Edinburgh arm 'ProDigit' elbow and shoulder sections, each joint controlled by a separate PIC18F4680, communicating along a CAN bus to a node at the distal end of the arm. This node also read the user intent from the joystick.

An arm that was clinically deployed for an extended period [15], has recently been replaced with the newer CAN based controllers, and a Mark 2 ProDigit elbow (all similar to arm above, referred to as 'the Clinical Arm'). The arm was constructed to test accelerometer compensation. An accelerometer was mounted with the wrist node in the wrist section so that the angle of the elbow and wrist relative to gravity could be recorded and sent along the arm to the forearm node and the input node.

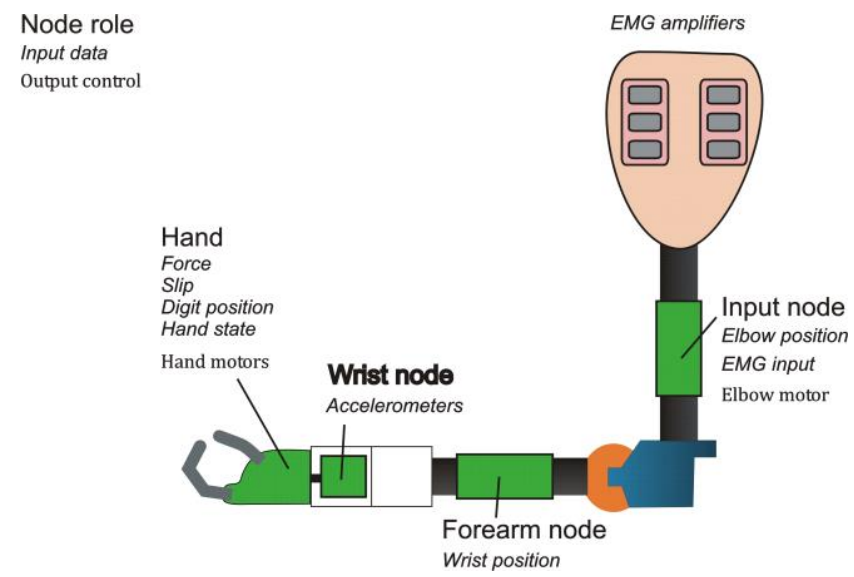

Fig. 4. Schematic of the ToMPAW 2 clinical arm

The arm consists of a socket, elbow unit, wrist unit and hand, (schematic, Figure 4, and an image of the completed arm Figure 5). A microprocessor controller in the humeral unit, acts as an input processor and takes in the signals from the user and the joints of the arm either directly, or via the CAN bus, and then instructs the arm and hand to move. The commands are: the state of the 
system (such as which joint should move), the digitised value of the EMG and some diagnostic information. Each node then reacts to the state of the arm moving their respective joints when required. The hand has a local controller that performs a hierarchical controller (SAMS, see later). The elbow is a ProDigit design from the Edinburgh Modular Arm System [16]. Its motor drives a worm and wheel and is positioned in the radial section of the arm. A microcontroller board is placed in the fore arm section to control the wrist. An accelerometer is positioned in the wrist section with a microprocessor board to convert the signals and transmit the data along the CAN bus to the master controller. The CAN bus links the arm controller to the wrist accelerometer. The digitised signal is smoothed using a running mean (length, 8 elements at $800 \mathrm{~Hz}$ ). The vector is then streamed to the master controller and the other nodes.

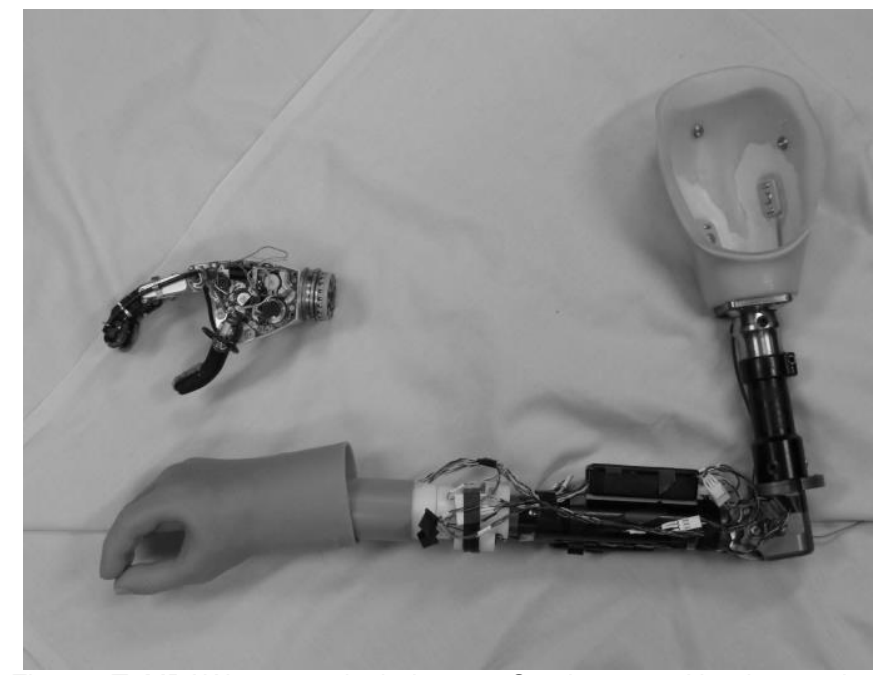

Fig. 5. ToMPAW 2 arm - includes two Southampton Hands, one has two degrees of freedom, the other a single degree based on a modified conventional commercial single axis hand.

A second role for the microprocessor board in the wrist is to convert the numerical command value of the EMG to an analogue signal which is passed to the hand controller via an industry standard wrist connector (Quick Disconnect Wrist - QDW). The conversion is required so that the user can swap out the advanced hand for other standard commercial hands. The advanced hand also has a CAN bus link and it communicates status information to the master controller.

\section{A. Southampton Adaptive Manipulation Scheme}

The hand is controlled using the Southampton Adaptive Manipulation Scheme (SAMS). This is a hierarchical control format; the user gives the hand simple instructions to open, close or hold and the controller adjusts the grip form and tension in response to the held object $[6,17]$. A SAMS hand moves through a state machine driven by the user's simple input instructions, from POSITION mode, where it is opened and closed to admit an object, through $\mathrm{TOUCH}$, when it attempts to apply the lightest contact, to HOLD, where sensors in the fingertips adjust the grip force to maintain a stable grip, by detecting the object slipping though the fingers. If the user wishes to override the reflex or apply greater tension a SQUEEZE state is invoked; here the drive motor voltage (and hence grip force) is proportional to the contraction level. Finally they can progress to a coordinated RELEASE phase. On this arm the hand had two independent degrees of freedom to perform tips opposition or power grip, automatically based on the initial point of contact between the hand and the target object [18].

When used in conjunction with the arm, the hand continues to operate hierarchically, it also reports back to the master controller when it is in HOLD mode. It is when this mode is selected that the motion compensation control of the wrist and elbow takes place. When the controllers receive the 'HOLD acknowledge' signal they perform gravity compensation, keeping the forearm and wrist at the same angle relative to the gravity vector at the time when the HOLD mode was invoked. If the operator selects the elbow or wrist axis to be controlled, then the joint is directly moved as before, but when the user selects a different axis, this new angle relative to gravity is now maintained. If the user invokes the SQUEEZE mode they wish to take over direct control of the grip and arm, and so the level compensation is turned off. Additionally, once the hand is instructed to RELEASE the object the angle relative to gravity is no longer maintained. These last two features are essential. Users do not wish to wear devices that do not behave in a predictable manner, moving when they don't expect it. So the correction must only take place when they have consciously selected it, in HOLD mode.

\section{B. Second example - two axis wrist}

A two degree of freedom wrist based on a differential, details of the device are in [19]. The two axes control both flexion/extension and pro/supination, thus they must both be driven whenever the wrist is moved. The output velocity, (or torque) from the wrist is thus the sum or difference of the two drive motors:

$$
\begin{aligned}
& V_{\text {flex }}=\left(V_{1}+V_{2}\right) \times R_{1} \\
& V_{\text {pronate }}=\left(V_{1}-V_{2}\right) \times R_{2}
\end{aligned}
$$

Where $R_{1}$ and $R_{2}$ are the reduction ratios of the differential with $V_{1}$ and $V_{2}$ as the velocities of the two motors. $V_{\text {flex }}$ and $V_{\text {pronate }}$ are the no load output velocities in the two directions. The wrist was driven serially, ie one biological axis at a time, but it still requires both motors to be run. The position of the two axes was detected using potentiometers on each axis. However, the orientation of the wrist can have a significant impact 
on how each motor has to be run, with or against, gravity.

Figure 6 shows the kinematics of the wrist. The output torque of the wrist is related to the two motors and their distance apart (d). A flexion moment comes from the combined center of mass of the hand and wrist distal to the joint. The pro/supination moment is generated by the combined center of mass not being in line with the axis of the wrist. The resulting torque from a hand on the end of the wrist is a torque from center of mass which both flexes and rotates the wrist.

To correct for the lever arm created by the hand, a triaxial accelerometer was placed in the wrist and its vector used to calculate the appropriate compensation for each of the drives to maintain the motion in one axis, irrespective of orientation of the wrist itself.

The direction of the wrist was calculated relative to the gravity vector and the motor drive was altered to counteract the torque of the hand on the end of the wrist. As the torque to pronate the wrist is proportional to the relative difference of the two motors, the correction was based on the relative change of the velocity demand for the motor:

$$
\begin{aligned}
& \text { Motor_1 }=V_{1} \times C_{1} \\
& \text { Motor_2 }=V_{2} / C_{2}
\end{aligned}
$$

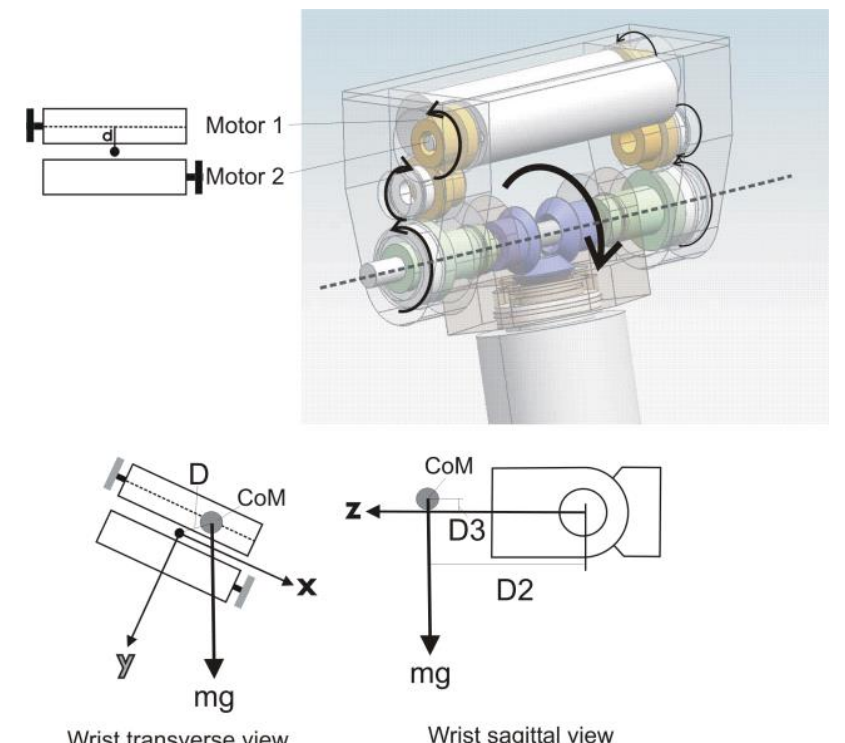

Fig. 6. Kinematics of the differential wrist. a) Wrist has two axes to drive a differential that drives flexion/extension and pro/supination. b) Shows the wrist flexing. c) The load imposed on the wrist by the offset mass of the hand creates a torque that needs to be resisted by the motors and is measured by the accelerometer. d) The torque is due to the mass of the hand on the end of the wrist.

Where Motors 1 and 2 are the motors in the wrist and $V_{1}$ and $V_{2}$ are the velocities of the two motors and $C_{1}$ and $C_{2}$ the correction factors for each motor respectively. With the ratios reversed if the wrist was inverted. Although one axis is driven at a time, in all cases it is the torque from the off axis load that causes the wrist to pronate or supinate, thus the correction is made against the rotation, at all times. Thus the lower motor is driven faster and the upper motor is slowed down, creating a torque proportional to the desired speed to oppose gravitational torque. $C_{1}$ and $C_{2}$ were found empirically.

\section{Demonstrations - Method}

To demonstrate the concepts the arms and wrists were built as described above. The tests described are the qualitative engineering demonstrations of the compensations. The clinical testing of the systems requires a different approach.

\section{A. Segment orientation}

Segment compensation aims to correct for the orientation of the joint and ensure the response to a user demand from the input can be uniform. To demonstrate this, the wrist was attached to a rigid mount and allowed to descend from fully flexed to fully extended.

This was repeated three times:

1/ Free fall, without any driven component the driver bridge set to open circuit.

2/ Driven full speed downwards.

3/ With Segment compensation on.

While the wrist was performing an extension towards gravity (hand moving downwards) the digitised value of the wrist flexion (as recorded by the potentiometer in the joint) was streamed along the bus to be recorded by the proximal node (conversation rate: $60 \mathrm{~Hz}$ ).

Initial testing of the clinical arm included mounting the humeral section on a handle. When the humerus was rigidly mounted hanging from a support, the elbow was driven with a signal equivalent to quarter full scale EMG to raise and lower the hand. This was performed five times, flexing extending over the full range of motion. The arm was either in a natural position so that flexion raised the hand, or the mounting of the arm was inverted, (as if over the head), so now elbow flexion would lower the hand with gravity.

\section{B. Motion compensation}

Also as part of the testing of the arm, the hand was used to grasp a cylindrical object and the HOLD mode was invoked to trigger the Motion Compensation. The humerus was then moved sideways to trigger the wrist to Pronate and Supinate to correct for the motion, or forward and backwards to trigger flexion and extension correction. 


\section{Results}

Figure 7 shows the motion as the wrist as it is driven downwards (darkest line), when it moves through free fall (medium line), and when the system controls the descent, with the additional information from the accelerometer. All data is taken from the potentiometer data and smoothed with a running mean over $66 \mathrm{~ms}$ (4 samples).

Table 1 summarizes the elbow performance as it flexes and extends with and against gravity. Despite widely different speeds uncorrected, corrected the motions were uniform to within $10 \%$ of each other.

\begin{tabular}{|l|l|l|l|}
\hline $\begin{array}{l}\text { Time of } \\
\text { motion }\end{array}$ & $\begin{array}{l}\text { No } \\
\text { correction }\end{array}$ & Correction & $\begin{array}{l}\text { Invert } \\
\text { arm }\end{array}$ \\
\hline $\begin{array}{l}\text { Motion down } \\
\text { (s) }\end{array}$ & $1.87 \pm 0.08$ & $2.64 \pm 0.07$ & $2.72 \pm 0.05$ \\
\hline Motion up (s) & $4.61 \pm 0.03$ & $2.71 \pm 0.06$ & $2.95 \pm 0.03$ \\
\hline
\end{tabular}

Table 1: Segment orientation - Performance of the elbow under gravity

Figures 8 and 9 show montages of stills from a video taken when the humeral section handle was moved.

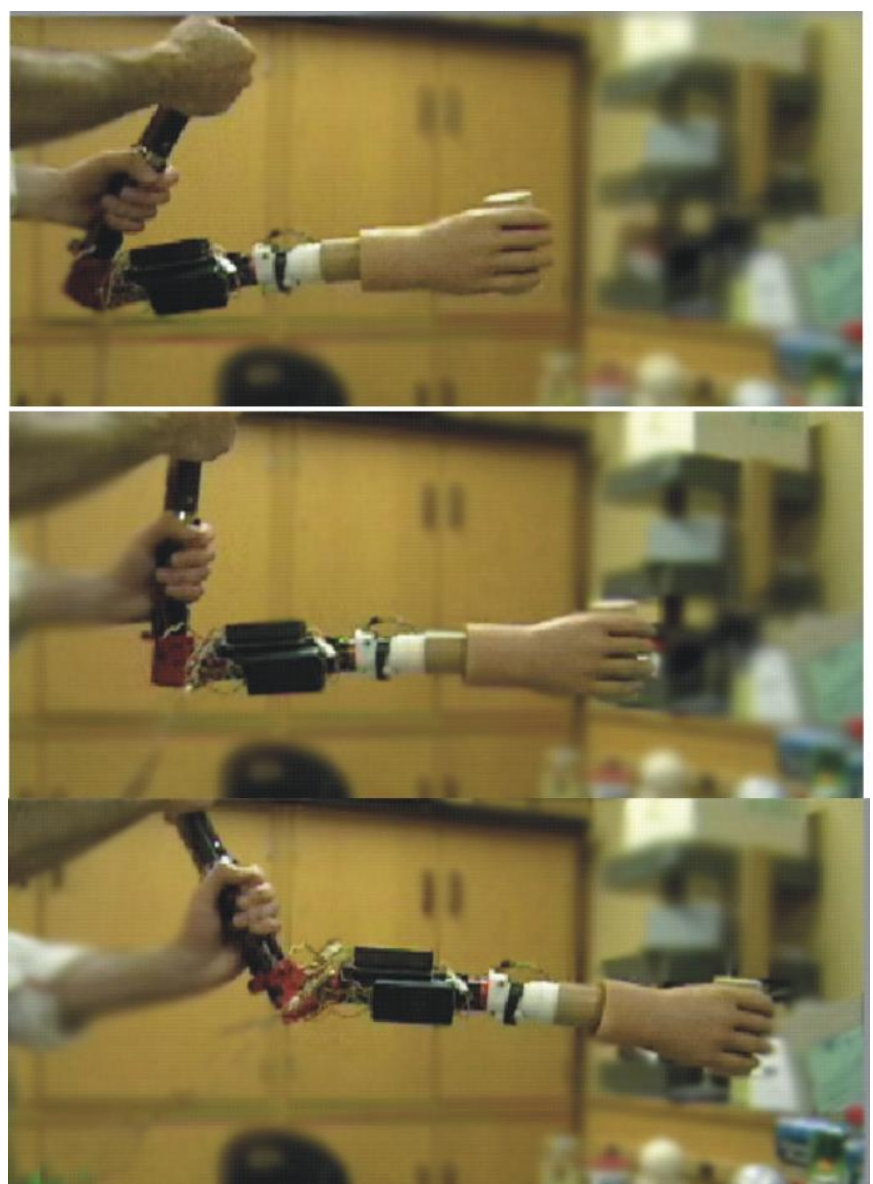

Fig. 9. Motion Compensation - The humeral section flexes and extends and the motion is detected by the accelerometers in the wrist and the elbow is driven to compensate for the motion.

\section{Discussion}

The aim of employing additional sensory information in the control of a prosthetic arm is to increase the ease of use and reduce the cognitive burden when using the arm. Most or all of the compensations could be performed by a skilled user without additional electronic sophistication, but this would make the arm more tiring to use, and hence less likely to be tolerated. By adding the knowledge of the orientation of the body segments as well as that of any held object the arm can

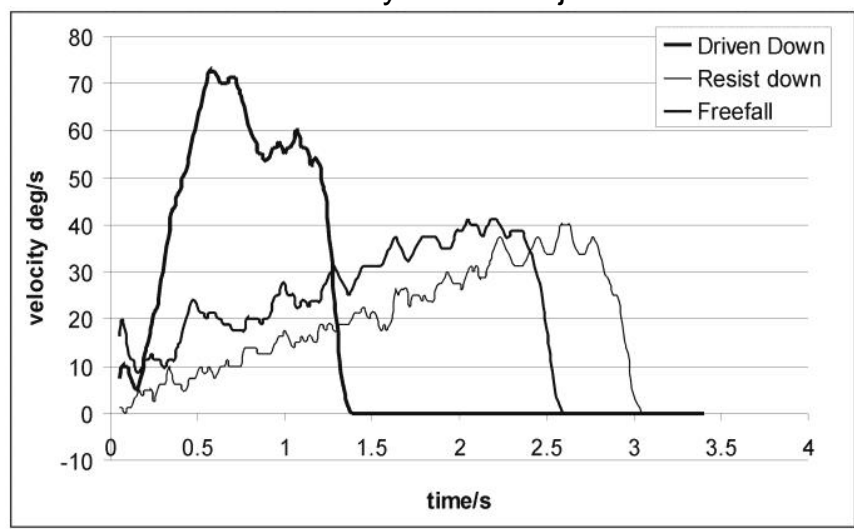

Fig. 7. Segment orientation - Flexion speed tests with differential wrist Chart shows the wrist if it is driven downwards, if it is allow to descend under gravity and if the controller maintains velocity control of the descent.

compensate for changes in the direction of gravity as the arm moves, as well as changes in body posture.

To demonstrate this the motion of the wrist was shown as it flexes downwards. If the wrist is driven by the motors it accelerates at $840{ }^{0} \mathrm{~s}^{-1}$. While the free fall of the wrist is $470{ }^{0} \mathrm{~s}^{-1}$. With the wrist in a controlled descent from the maximum EMG joint velocity demand it is $180^{0} \mathrm{~s}^{-1}$.

The test of the elbow shows the impact on the acceleration of the hand as it flexes downwards. For this the motion of the elbow must be predictable, that is, that for a given level of muscular effort to command the hand to raise or lower, the speed of motion should be the same, with or without gravity, however the arm is held. The results show that although there is roughly two and a half times difference between the flexing up and extending down in the uncontrolled arm, when the gravity compensation is added there is a $3 \%$ difference. Even with the arm inverted the difference remains a few percent.

Additional clinical testing of the entire ToMPAW2 system is being conducted with the user of the earlier system. Such testing is appropriate for reporting in a clinical journal. 


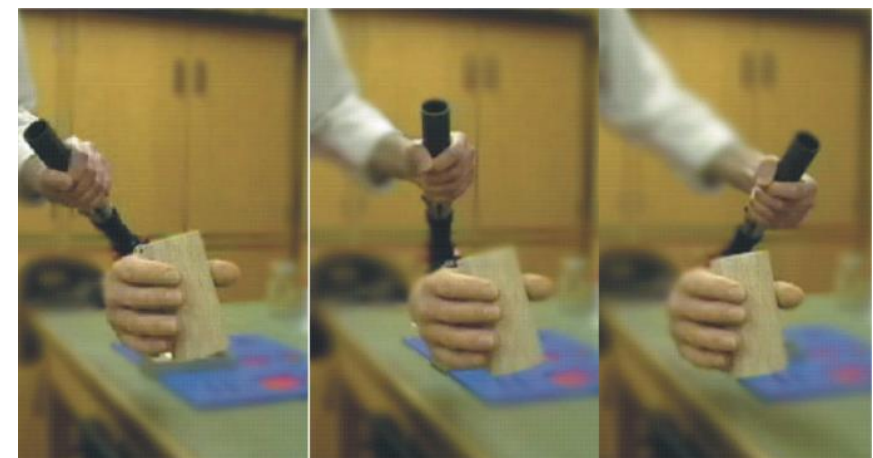

Fig. 8. Motion Compensation - The humeral section is rotated in a similar manner to humeral ab/adduction and the motion is detected by the accelerometers in the wrist and the wrist is driven to compensate for the motion.

\section{CONCLUSION}

Accelerometers can be used to supply additional information to assist in the control of prosthetic arms. They can be used to measure the orientation of the segments and allow the controller to automatically maintain the orientation of the distal segments, as well as correct the input demand from the user so that it is as easy for the user to raise the arm against gravity as lower the hand with gravity.

\section{ACKNOWLEDGEMENTS}

The authors would like to thank various organizations that have supported this work: Canadian Foundation for Innovation, Canada Institutes of Health Research, New Brunswick Innovation Foundation.

\section{REFERENCES}

[1] M Flanders, "What is the biological basis of sensorimotor integration?" Biological Cybernetics vol.104 (1-2), pp. 1-8, 2011.

[2] E.Nebot and H.Durrant-Whyte, "Initial calibration and alignment of low cost inertial navigation units for land vehicle applications." Journal of Robotics Systems, vol.16 (2), pp. 81-92, February 1999.

[3] I.D. Swain and J.M. Nightingale, "An adaptive control system for a complete hand/arm prosthesis." Journal of Biomedical Engineering, vol.2:163-166, July 1980.

[4] I.D. Swain, "Adaptive control of an arm prosthesis." PhD thesis, Electrical Engineering Department, University of Southampton, Southampton, Hampshire, 1982.

[5] N. Storey, R.W. Todd, and J.M. Nightingale, "Cartesian co-ordinate control of a complete arm prosthesis." in Proceedings of the 5th International Symposium on External Control of Human Extremities, Dubrovnik, Yugoslavia, August 25-30, 1975, pp. 489497.

[6] J.M. Nightingale, "Microprocessor control of an artificial arm." Journal of Microcomputer Applications, vol.8:167-173, 1985.

[7] P.J. Kyberd, A. S. Poulton, L. Sandsjö, S. Jönsson, B. Jones, and D. Gow, "The ToMPAW modular prosthesis - A platform for research in Upper Limb prosthetics." Journal of Prosthetics and Orthotics, vol.19(1):12-21, January 2007.
[8] L. Miller, K. Stubblefield, S. Finucane, R. Lipschutz, and T. Kuiken, "A comparison of direct control and pattern recognition control of a seven degree-of-freedom hand wrist system." in ISPO 2013 World Congress, p. 346. International Society of Prosthetics and Orthotics, Hyderabad, India, 4th - 7th February 2013.

[9] A. Fougner, E. Scheme, A.D.C Chan, K. Englehart and $\varnothing$. Stavdahl, "Resolving the Limb Position Effect in Myoelectric Pattern Recognition." IEEE Transactions on Neural Systems and Rehabilitation Engineering, vol.19(6): 644 - 651, DOI 10.1109.

[10] T.A. Kuiken, G.A. Dumanian, R.D. Lipschutz, L.A. Miller, and K.A. Stubblefield, "The use of targeted muscle reinnervation for improved myoelectric prosthesis control in a bilateral shoulder disarticulation amputee." Prosthetics and Orthotics International, vol.28(3):245-253, December 2004.

[11] A. Zinck, "The investigation of compensatory movements in prosthesis users and the design of a novel wrist." Masters, Department of Mechanical Engineering, University of New Brunswick, Fredericton, New Brunswick, 2008.

[12] A. Muzumdar (Ed) Powered Upper Limb Prosthetics, Springer, 2004, ISBN 3-540-40406-6.

[13] F. Popa, P. Kyberd, A. Hussani, P. Gosine and W. Hill, "Assessment of Prosthesis Use by Visual Attention Analysis." presented in TIPS'12 Trent International Prosthetics Symposium, Loughborough, Leicestershire, UK, 21-24 May 2012.

[14] A. Poulton, P.J. Kyberd, and D. Gow, "Progress of a Modular Prosthetic Arm", Chapter 19, in Universal Access and Assistive Technology, pages 193-200. Springer, London, 2002. (S. Keates et al Editors)

[15] A. Poulton and P.J. Kyberd, "Evaluation of a modular prosthetic arm." presented TIPS'12 Trent International Prosthetics Symposium, Loughborough, Leicestershire, UK, 21-23 May 2012.

[16] D. Gow, W. Douglas, C. Geggie, E. Monteith, and D. Stewart, "The development of the Edinburgh Modular Arm System." Proceedings of the Institution of Mechanical Engineers Part H, 215:291-298, 2001.

[17] P.J. Kyberd and P.H. Chappell, "The Southampton Hand: An Intelligent Myoelectric Prosthesis." Journal of Rehabilitation Research and Development, vol.31(1):326-334, 1994.

[18] P.J. Kyberd, M. Evans, and S. te Winkel, "An intelligent anthropomorphic hand, with automatic grasp." Robotica, vol.16:531-536, 1998.

[19] P.J.Kyberd, E.Lemaire, E.Scheme, L.Goudreau, G.Bush, W.Hill, C.MacPhail, M.Brookeshaw, and T.Scribner, "A two degree of freedom powered prosthetic wrist." Journal of Rehabilitation Research and Development, vol.48(6):609-618, 2011.

Peter J. Kyberd received the Ph.D. degree in the control of a multifunction prosthetic hand from

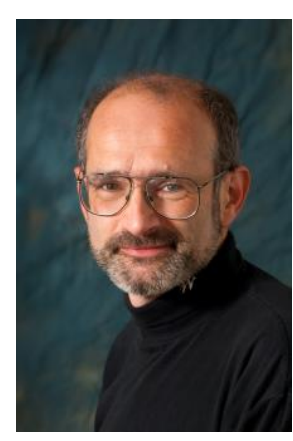
Southampton University, Southampton, U.K. Putting the first ever microprocessor controlled hand in the field for trials with a user.

During the 1990s, he worked at the Oxford Orthopaedic Engineering Centre, where he was involved in a verity of orthopaedic projects including two projects funded by the European Union investigating aspects of the design and 
control of prosthetic arms. With Dr Poulton he fitted the first prosthetic limb to be controlled by a serial bus and used in the field in 2000.

From 2000, he was a Lecturer at the Cybernetics Department of Reading University in the U.K. where he was part of a team that performed the first implant of a bi-directional nerve sensor on a healthy human being.

In 2003, he took up a Canada Research Chair in Rehabilitation Cybernetics at the Institute of Biomedical Engineering, where he conducted research in the clinical application of intelligent prosthetic arms.

In 2015 he became the Head of the Engineering Science Department of Greenwich University in the UK.

Adrian Poulton received the $\mathrm{PhD}$ degree in Electrical Engineering applied to Medicine from Imperial College, London, UK in 1979.

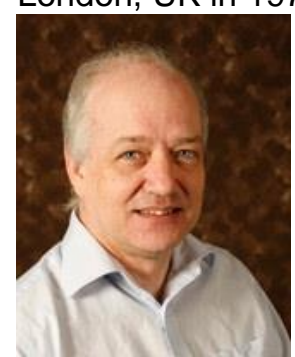

$\mathrm{He}$ worked at the Oxford Orthopaedic Engineering Centre in the late 1990's and is currently a lecturer at The Open University, Milton Keynes, UK. His research interests include prosthetics, rehabilitation and communications.

With Dr Kyberd he fitted the first prosthetic limb to be controlled by a serial bus and used in the field in 2000. 